\title{
Prevalence of Overweight and Obesity in Patients with Spinal Cord Injury
}

\author{
Seyedeh Samaneh Esteghamat Hanzai ${ }^{1}$, Sanaz Moghadami Monaghi ${ }^{1}$, Ali Gorji ${ }^{*}$ \\ ${ }^{1}$ Shefa Neuroscience Research Center, Khatam Alanbia Hospital, Tehran, Iran. \\ ${ }^{2}$ Epilepsy Research Center, Westfälische Wilhelms-Universität Münster, Münster, Germany.
}

\section{ABSTRACT}

Introduction: Obesity is one of the most prevalent diet-related problems and increases the risk for diabetes mellitus, hypertension, atherosclerosis, and dyslipidemia. Cardiovascular diseases are one of the major of morbidity and mortality in people with a spinal cord injury (SCI). Increased fat mass has also been identified as an important risk factor in chronic SCI and weight management is recommended as an important preventive strategy of cardiovascular disease. The aim of this study is to determine the prevalence of overweight and obesity in people with SCI. Materials and Methods: An observational analytic cross sectional study was done on 850 SCI patients registered in Khatam Alanbia Hospital, Tehran, Iran, from 2008 to 2013. These data are included age, sex, height, weight, duration of SCI, and the level of SCI. The body mass index (BMI) was subsequently calculated for each patient and the prevalence of overweight and obesity were determined. Results: Of the 850 patients, 833 patients were male and 17 were female. In all, $50.2 \%$ of patients had a normal BMI and $7.6 \%$ of patients were undernourished. The prevalence of overweight and obesity were $32.1 \%$ and $10 \%$, respectively. There was a significant relation between age and body mass index but there was no significant correlation between the level of injury and body mass index. Conclusion: Prevalence of overweight and obesity are high in people with SCI and there is a significant positive correlation between BMI and age.

\section{Key words:}

1. Spinal Cord Injuries

2. Obesity

3. Body Mass Index

\section{* Corresponding Author: Ali Gorji}

E-mail:gorjial@uni-muenster.de 


\title{
شيوع اضافه وزن و جاقى در بيماران آسيب نخاعى
}

\author{
سيده سمانه استقامت هنزائى'، ساناز مقدمى مونقى'، على كرجى" \\ 'مركز تحقيقات علوم اعصاب شفا، بيمارستان خاتم الانبياء، تهران، ايران. \\ †مركز تحقيقات صرع، دانشكاه مونستر، مونستر، آلمان.
}



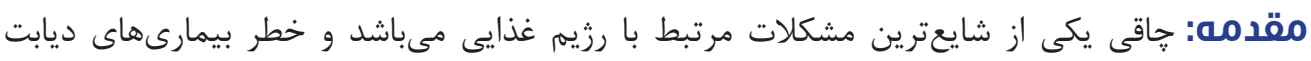

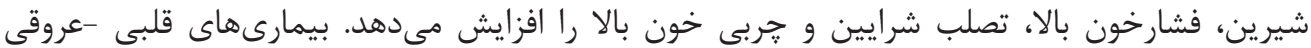

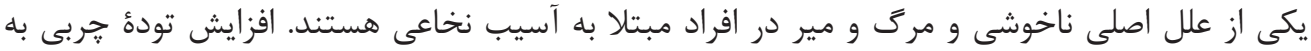

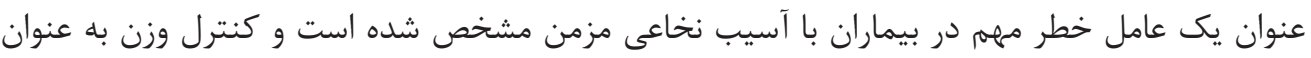

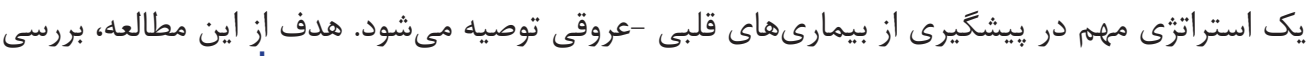



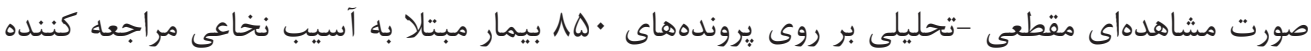

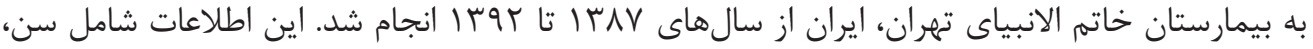





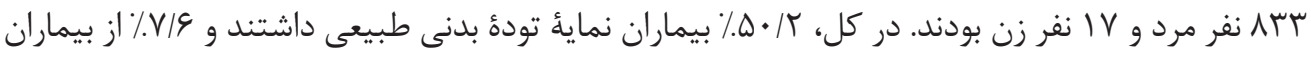



كليد وازمها:

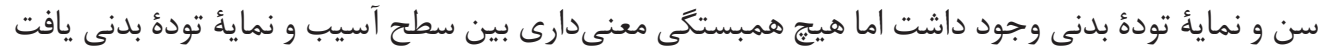



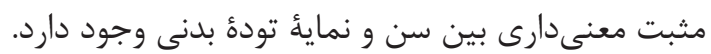

ا. آسيبهاى طناب نخاعى r. r.
" نويسنده مسئول: على گرجى : آدرس الكترونيكى: gorjial@uni-muenster.de 


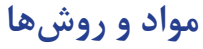

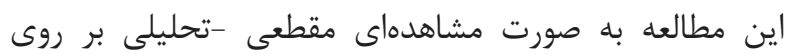



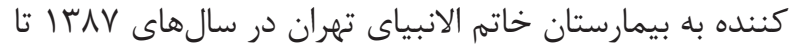

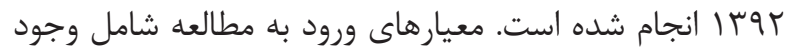

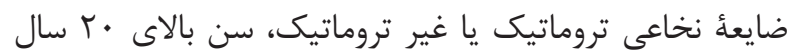

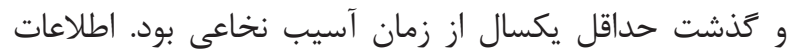

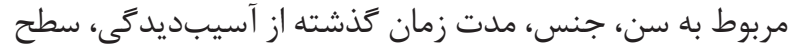

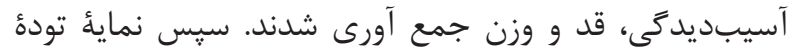

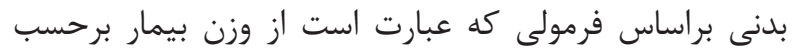

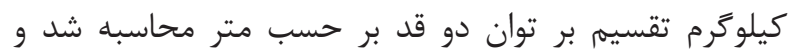

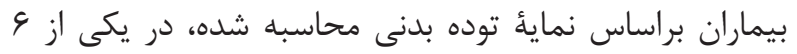

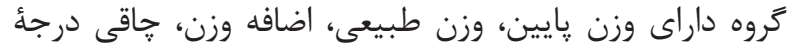

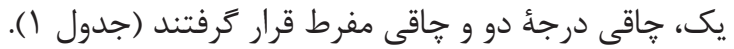
تمامى دادههاى به دست آمده در بانك اطلاعاتى رايانهاى

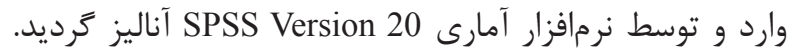

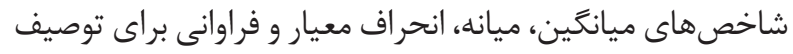

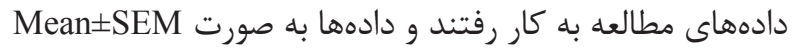

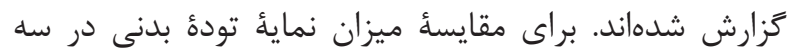

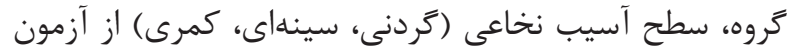

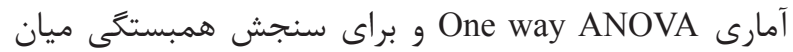

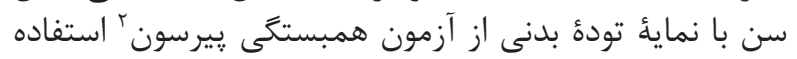

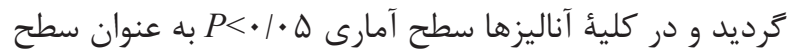
معنى دارى در نظر كرفته شد.

بافته ها

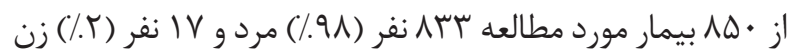





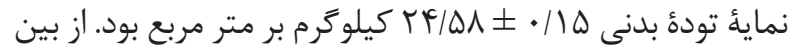

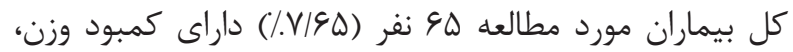



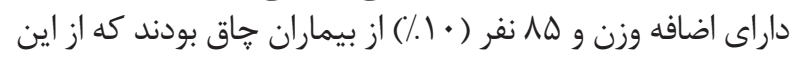

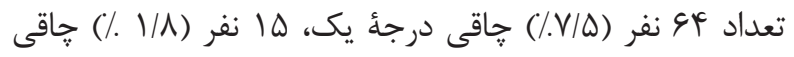

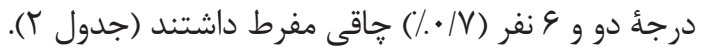

مقايسٔ نمائُ تودهٔ بدنى در سه كروه سطح آسيب نخاعى توسط

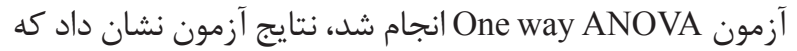

جاقى يكى از شايعترين مشكلات مرتبط با رزيم غذايى در جهان

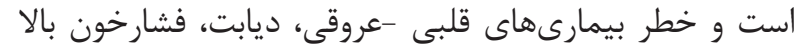



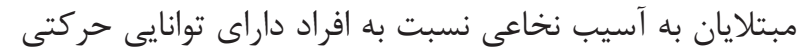

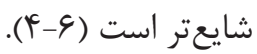

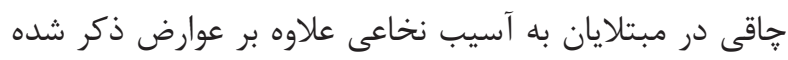

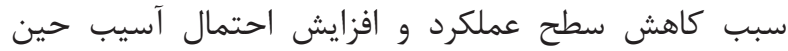
مراقبتهاى يرستارى مىشود (V-9)





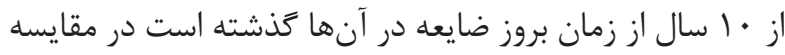
با افراد سالم با سن برابر، شيوع بيشترى دارد (ه) (ه).

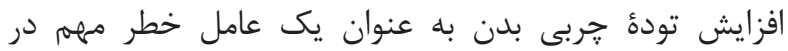

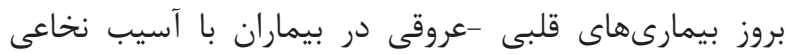

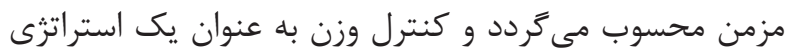



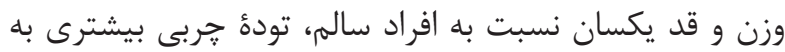

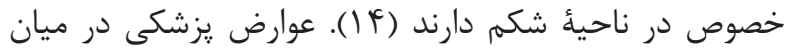

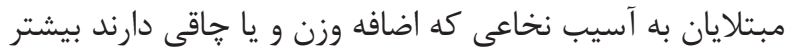

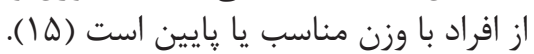

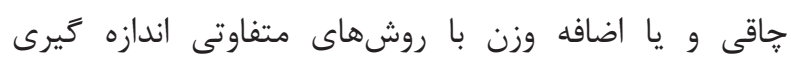

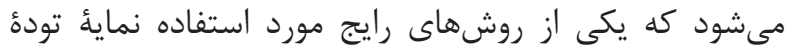

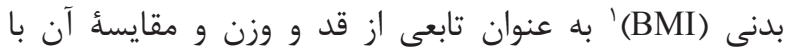

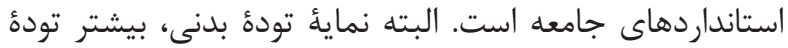



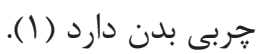



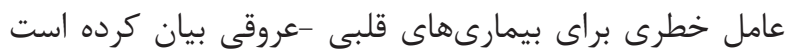

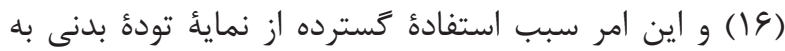



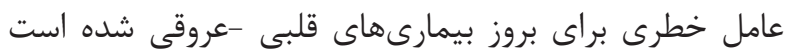

هدف از اين مطالعه بررسى ميزان شيوع جاقى و اضافه وزن در

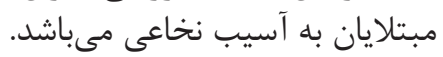

جدول ا- طبقه بندى سازمان جهانى بهداشت براى نمائُ تودة بدنى در بالغين (19).

\begin{tabular}{|c|c|}
\hline ميزان نمايئ توده بدنى & طبقه بندى \\
\hline كمتر از N/D & كمبود وزن \\
\hline TF/99 تا IN/D & وزن طبيعى \\
\hline rq/৭q تا rd & اضافه وزن \\
\hline rr/qq r r & جاقى درجلَ يك \\
\hline ґq/৭q ته & جاقى درجأ دو \\
\hline بيشتر از • ب & جاقى مفرط \\
\hline
\end{tabular}

${ }^{1}$ Body mass index (BMI)

${ }^{2}$ Pearson 
جدول ץ- فراوانى كروههاى نمائ تودة بدنى.

\begin{tabular}{|c|c|c|}
\hline درصد & فراوانى & كروههاى نمايهُ تودهُ بدنى \\
\hline$V / \& D$ & 90 & كمبود وزن \\
\hline$\Delta \cdot 10$ & GTV & وزن طبيعى \\
\hline$T r / l$ & rVT & اضافه وزن \\
\hline 1 & $\Lambda \Delta$ & جاقى \\
\hline
\end{tabular}



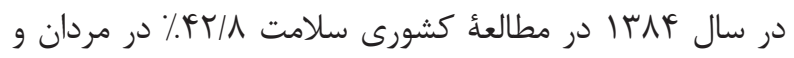

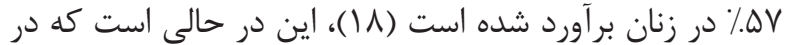

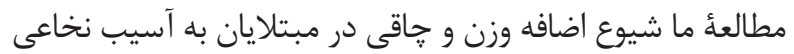

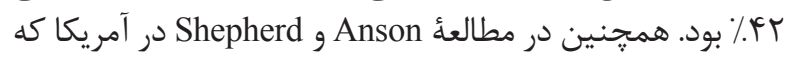

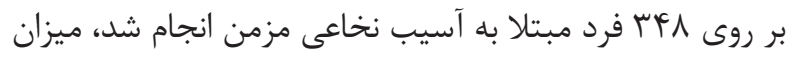

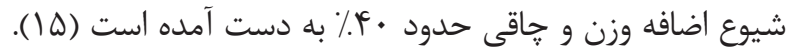



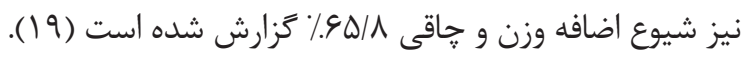

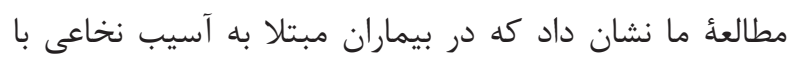

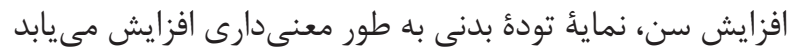

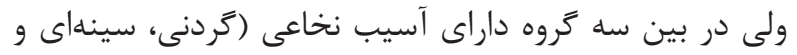

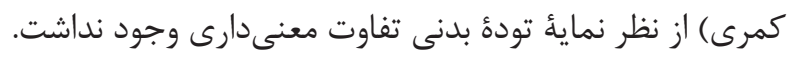

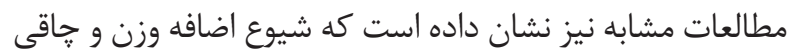

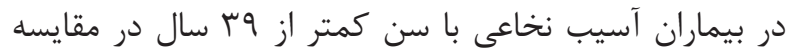

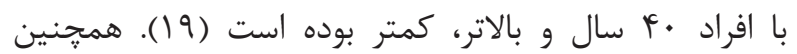

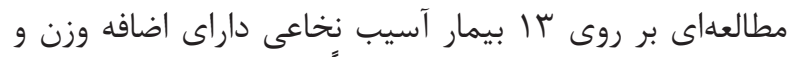

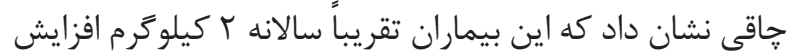

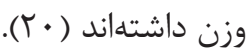

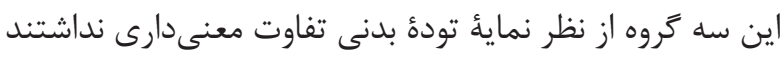

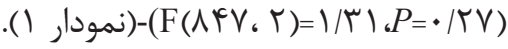

طبق نتايج آزمون همبستخى يِيرسون ارتباط معنى دارى بين

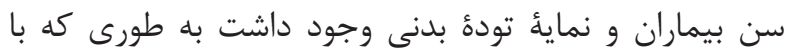

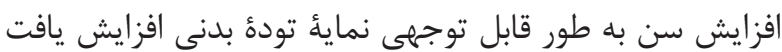
. $(P<\cdot / \cdot \Delta ، \mathrm{~N}=\Lambda \Delta \cdot$ ، $\mathrm{r}=\bullet / /)$

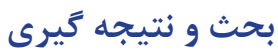

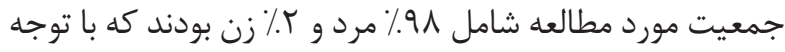


نسبت بالاى مرد به زن قابل انتظار است.

تاقى يكى از شايعترين مشكلات مرتبط با تغذيه است. نمائ

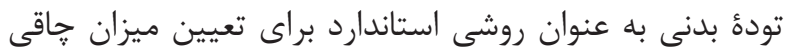

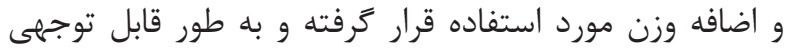

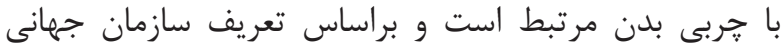

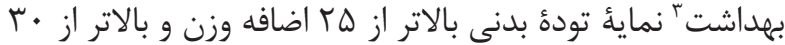

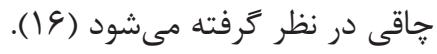
در بررسى هاى انجام شده در ايران، فراوانى اضافه وزن و جاقى تردي 
با توجه به اين كه در حال حاضر بيمارىهاى قلبى -عروقى علت

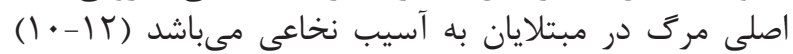

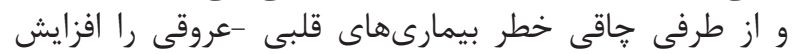

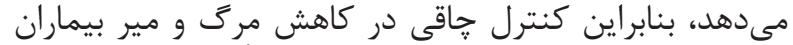

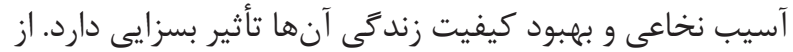

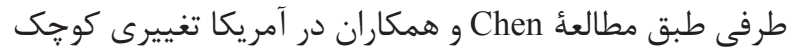

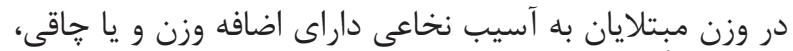

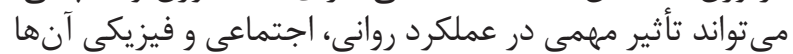

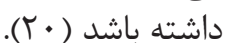

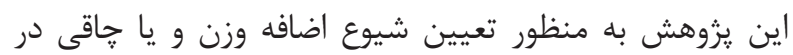

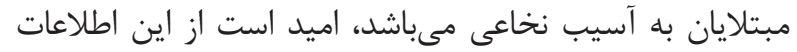

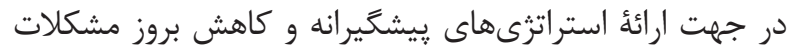

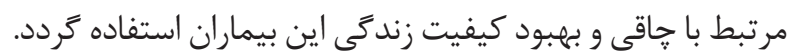

1. Everett Koop C. The surgeon general's report on nutrition and health: summary and recommendations. DIANE Publishing. 1994.

2. Ernst ND, Obarzanek E, Clark MB, Briefel RR, Brown $\mathrm{CD}$, Donato $\mathrm{K}$. Cardiovascular health risks related to overweight. J Am Diet Assoc. 1997; 97(7): S47-S51.

3. Brown CD, Higgins M, Donato KA, Rohde FC, Garrison R, Obarzanek E, et al. Body mass index and the prevalence of hypertension and dyslipidemia. Obes Res. 2000; 8(9): 605-19.

4. Bauman W, Spungen A. Carbohydrate and lipid metabolism in chronic spinal cord injury. J Spinal Cord Med. 2000; 24(4): 266-77.

5. Imai K, Kadowaki T, Aizawa Y, Fukutomi K. Morbidity rates of complications in persons with spinal cord injury according to the site of injury and with special reference to hypertensio. Paraplegia. 1994; 32(4): 246-52.

6. Yekutiel M, Brooks M, Ohry A, Yarom J, Carel R. The prevalence of hypertension, ischaemic heart disease and diabetes in traumatic spinal cord injured patients and amputees. Paraplegia. 1989; 27(1): 58-62.

7. Brentin L, Sieh A. Caring for the morbidly obese. Am J Nurs. 1991; 91(8): 40-3.

8. Hudelson E. Points to remember when caring for an obese patient. Nursing. 1992; 22: 62-3.

9. Blackmer J, Marshall S. Obesity and spinal cord injury: an observational study. Spinal Cord. 1997; 35(4): 245-7.

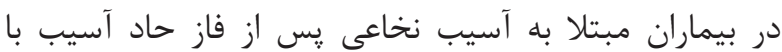

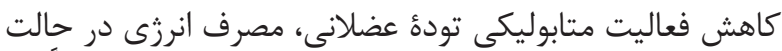



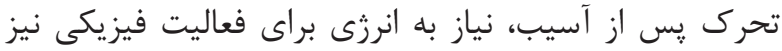

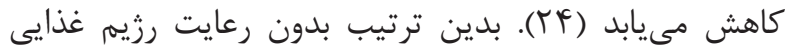

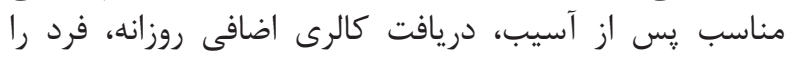

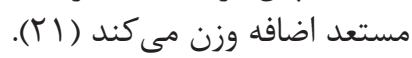

جاقى و اضافه وزن خطر بيمارىهاى قلبى -عروقى، ديابت،

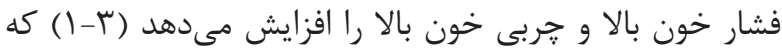

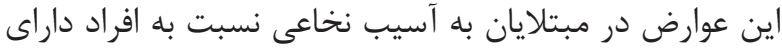



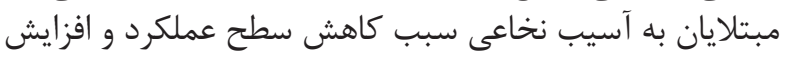
احتمال آسيب حين مراقبتهاى يرستارى مىشود (Y-9).

$$
\text { منابع }
$$

10. Hartkopp A, Brønnum-Hansen H, Seidenschnur AM, Biering-Sørensen F. Survival and cause of death after traumatic spinal cord injury. A long-term epidemiological survey from Denmark. Spinal Cord. 1997; 35(2): 76-85.

11. De Vivo MJ, Black KJ, Stover SL. Causes of death during the first 12 years after spinal cord injury. Arch Phys Med Rehabil. 1993; 74(3): 248-54.

12. Whiteneck GG, Charlifue SW, Frankel HL, Fraser MH, Gardner BP, Gerhart KA, et al. Mortality, morbidity, and psychosocial outcomes of persons spinal cord injured more than 20 years ago. Paraplegia. 1992; 30(9): 617-30.

13. Sabharwal S. Cardiovascular dysfunction in spinal cord disorders. Spinal Cord Medicine-Principles and Practice. New York, NY; Demos Publishing. 2003; p. 179-92.

14. Jones LM, Legge M, Goulding A. Healthy body mass index values often underestimate body fat in men with spinal cord injury. Arch Phys Med Rehabil. 2003; 84(7): 1068-71.

15. Anson CA, Shepherd C. Incidence of secondary complications in spinal cord injury. I Int J Rehabil Res. 1996; 19(1): 55-66.

16. World Health Organization. Obesity: preventing and managing the global epidemic: Report of a WHO Consultation. WHO Library Cataloguing-in-Publication Data. 2000.

17. NOEIE Panel. Clinical guidelines on the identification, evaluation, and treatment of overweight 
and obesity in adults. 1998.

18. Finucane MM, Stevens GA, Cowan MJ, Danaei G, Lin JK, Paciorek CJ, et al. National, regional, and global trends in body-mass index since 1980: systematic analysis of health examination surveys and epidemiological studies with 960 country-years and 9.1 million participants. Lancet. 2011; 377(9765): 557-67.

19. Gupta N, White K, Sandford P. Body mass index in spinal cord injury-a retrospective study. Spinal Cord. 2005; 44(2): 92-4.

20. Chen Y, Henson S, Jackson AB, Richards JS. Obesity intervention in persons with spinal cord injury. Spinal Cord. 2005; 44(2): 82-91.

21. Cox SA, Weiss SM, Posuniak EA, Worthington
P, Prioleau M, Heffley G. Energy expenditure after spinal cord injury: an evaluation of stable rehabilitating patients. J Trauma. 1985; 25(5): 419-23.

22. Buchholz AC, Mc Gillivray CF, Pencharz PB. Differences in resting metabolic rate between paraplegic and able-bodied subjects are explained by differences in body composition. Am J Clin Nutr. 2003; 77(2): 371-8.

23. Monroe MB, Tataranni PA, Pratley R, Manore MM, Skinner JS, Ravussin E. Lower daily energy expenditure as measured by a respiratory chamber in subjects with spinal cord injury compared with control subjects. Am J Clin Nutr. 1998; 68(6): 1223-7.

24. Buchholz AC, Mc Gillivray CF, Pencharz PB. Physical Activity Levels Are Low in Free-Living Adults with Chronic Paraplegia. Obes Res. 2003; 11(4): 563-70. 